\title{
Efeitos das mudanças do uso da terra na biogeoquímica dos corpos d'água da bacia do rio Ji-Paraná, Rondônia.
}

\author{
Alex Vladimir KRUSCHE ${ }^{1 *}$; Maria Victoria Ramos BALLESTER ${ }^{1}$; Reynaldo Luiz VICTORIA ${ }^{1}$; Marcelo Correa \\ BERNARDES $^{1}$; Nei Kavaquichi LEITE $^{1}$; Laís HANADA ${ }^{1}$; Daniel de Castro VICTORIA ${ }^{1}$; André Marcondes \\ TOLEDO $^{1}$; Jean Pierre OMETTO ${ }^{1}$; Marcelo Zacharias MOREIRA ${ }^{1}$; Beatriz Machado GOMES $^{2}$; Marcos Alexandre \\ BOLSON $^{2}$; Sérgio Gouveia NETO ${ }^{2}$; Nilton BONELLI ${ }^{3}$; Linda DEEGAN ${ }^{4}$; Christopher NEILL ${ }^{4}$; Suzanne \\ THOMAS $^{4}$; Anthony Keith AUFDENKAMPE5; Jeffrey Edward RICHEY 5 .
}

\begin{abstract}
RESUMO
Este trabalho discute os efeitos das mudanças do uso do solo na biogequímica dos rios da bacia de drenagem do rio Ji-Paraná (Rondônia). Nesta região, a distribuição espacial do desmatamento e das propriedades do solo resultam em sinais diferentes, possibilitando a divisão dos sistemas fluviais em três grupos: rios com águas pobres em íons e baixo impacto; rios com conteúdo iônico intermediário e impacto médio e rios com elevados conteúdo iônico e impacto antropogênico. As características biogeoquímicas dos rios têm relação significativa com a área de pasto, melhor parâmetro para prever a condutividade elétrica $\left(\mathrm{r}^{2}=0,87\right)$ e as concentrações de sódio $\left(\mathrm{r}^{2}=\right.$ $0,75)$, cloreto $\left(r^{2}=0,69\right)$, potássio $\left(r^{2}=0,63\right)$, fosfato $\left(r^{2}=0.78\right)$, nitrogênio inorgânico $\left(r^{2}=0.52\right)$, carbono inorgânico $\left(r^{2}=0.81\right)$ e carbono orgânico (rain ${ }^{2}=0.51$ ) dissolvidos. Cálcio e magnésio tiveram sua variância explicada pelas características do solo e pastagem. Nossos resultados indicam que as mudanças observadas na micro-escala constituem "sinais biogeoquímicos" gerados pelo processamento do material nas margens dos rios. A medida em que os rios evoluem para ordens superiores, os sinais persistentes nos canais fluviais estão mais associdados às características da bacia de drenagem (solos e uso da terra). Apesar dos efeitos das mudanças observadas no uso do solo não serem ainda detectáveis na macro-escala (bacia amazônica), a disrupção da estrutura e funcionamento dos ecossistemas é detectável nas micro e meso escalas, com alterações significativas na ciclagem de nutrientes nos ecossistemas fluviais.
\end{abstract}

\section{PALAVRAS-CHAVE}

Amazônia, rios e igarapés, biogeoquímica, mudanças no uso da terra.

\section{Effects of land use changes in the biogeochemistry of fluvial systems of the Ji-Paraná river basin, Rondônia.}

\begin{abstract}
In this article we present the results of the effects of land use change on the river biogeochemistry of the Ji-Paraná basin (Rondônia). In this region, the spatial distribution of deforestation and soil properties result in different biogeochemical signals, allowing the division of the fluvial systems into three groups: rivers with low ionic concentration and low impact; rivers with intermediate ionic content and medium impact; and rivers with high ionic content and anthropogenic impact. River biogeochemical characteristics present a significant correlation with pasture area, the best predictor for electric conductivity $\left(r^{2}=0,87\right)$, sodium $\left(r^{2}=0,75\right)$, chloride $\left(r^{2}=0,69\right)$, potassium $\left(r^{2}=0,63\right)$, phosphate $\left(r^{2}=0,78\right)$, and dissolved inorganic nitrogen $\left(r^{2}=0,52\right)$, inorganic carbon $\left(r^{2}=0,81\right)$ and organic carbon $\left(r^{2}=0,51\right)$. For calcium and magnesium, both soil properties and pasture explained most of the observed variability. Our results indicate that the changes observed at the micro-scale constitute "biogeochemical signals" generated by the material processing at the riparianzones. As the rivers evolve to higher orders, the persistent signals in the fluvial channels are very closely related to the drainage basin characteristics (soils and land use), which, in turn, become the determinant of these systems dynamics. While at the macro-scale (the whole basin) the effects of land use changes are notyet detectable in the Amazon, the disruption of the structure and functioning is occuring at the micro and meso scales, with significant alterations of nutrient cycling in fluvial ecosystems
\end{abstract}

\section{KEYWORDS}

Amazonia, Rivers and streams, biogeochemistry, land-use change

${ }^{1}$ Centro de Energia Nuclear na Agricultura. Av. Centenário, 303. 13416-00. Piracicaba, SP, Brasil.

${ }^{2}$ Universidade Federal de Rondônia. Estrada do Itapirema s/n. Ji-Paraná, RO, Brasil.

${ }^{3}$ Universidade Luterana do Brasil. Av. Universitária, 762. Ji-Paraná, RO, Brasil.

${ }^{4}$ Marine Biological Laboratory. 7 MBL Street. Woods Hole, MA, EUA.

${ }^{5}$ University of Washington. School of Oceanography, Seattle WA 98112. EUA 


\section{ACTA \\ AMAZONICA}

EFEITOS DAS MUDANÇAS DO USO DA TERRA NA BIOGEOQUÍMICA

DOS CORPOS D'ÁGUA DA BACIA DO RIO JI-PARANÁ, RONDÔNIA.

\section{INTRODUÇÃO}

A Amazônia é um dos mais importantes ecossistemas do planeta, devido à extensão de suas florestas tropicais úmidas, à biodiversidade que estas contém e às taxas em que estão sendo modificadas. Dos cerca de 5 milhões de $\mathrm{km}^{2}$ de floresta, mais de $587 \mathrm{mil} \mathrm{km}^{2}$ já foram desmatados apenas no território brasileiro (Brasil, 2002), principalmente devido ao desenvolvimento rápido ocorrido a partir da década de 70 .

Outra característica fundamental da região amazônica é sua extensa rede de drenagem, a qual culmina no maior rio do mundo (Richey et al., 1980). Ao longo do seu canal principal e dos tributários, existem entre 100.000 a $300.000 \mathrm{~km}^{2}$ de áreas periodicamente inundadas (Sippel et al., 1994; Junk, 1997), enquanto as zonas ribeirinhas dos pequenos igarapés podem abranger em torno de 1 milhão de $\mathrm{km}^{2}$. Contudo, apenas recentemente a importância destas áreas na ciclagem regional e global de nutrientes começou a ser melhor reconhecida. Richey et al., (2002) demostraram que estas áreas podem ter um papel fundamental na ciclagem regional e global de carbono. Rios e áreas inundáveis da região central da bacia exportam, através da evasão de $\mathrm{CO}_{2}$, em torno de $1,2 \pm 0,3 \mathrm{MgCha}^{-1}$ ano ${ }^{-1}$, constituindo um fluxo de saída de $0,5 \mathrm{Gt}^{-a n 0^{-1}}$ de carbono na bacia. Este carbono provavelmente é gerado pela decomposição da matéria orgânica originária das florestas de terra firme e inundáveis, o qualé respirado nos rios e várzeas e liberado para a atmosfera na forma gasosa. Consequentemente, pode-se assumir que os compartimentos terrestre e aquático do ecossistema amazônico estão intimamente acoplados, e as mudanças no primerio podem afetar drasticamente a dinâmica do segundo.

O uso e a cobertura do solo têm um papel fundamental no delineamento do ambiente em escalas global, regional e local. Mudanças no uso e cobertura do solo influenciam a diversidade biológica (Sala et al., 2000), o clima (Gash et al., 1996) e os ciclos biogeoquímicos e da água (Meixner \& Eugster, 1999). A retirada da floresta nativa pode resultar em aumentos da temperatura do solo, da erosão, e em modificações do balanço hídrico e na disponibilidade de nutrientes (Vitousek \& Melillo 1979; Leopoldo et al, 1987; Swank \& Crossley, 1988). Consequentemente, o transporte de sedimentos, material orgânico e nutrientes associados para os rios é também alterado (Hunsaker \& Levine, 1995; Sharpley et al., 1995; Meyer et al., 1988). Entender como os processos e os controles ambientais variam entre florestas nativas, planíceis de inundação e áreas agrícolas e de rebrota é crucial para identificar os fatores que poderno limitar o desenvolvimento de usos do solo específicos.

$\mathrm{Na}$ Amazônia, os estudos já efetuados descrevem de forma extensa a hidrologia e biogeoquímica do canal principal do rio Amazonas e seus tributários mais importantes (Stallard \& Edmond, 1987; Richey et al., 1990; 1991; Richey \& Victoria, 1993; entre outros), bem como dos lagos de várzea da planície central (Sioli, 1984; Melack \& Fisher, 1990; Lesack \& Melack, 1995). Nesta escala (macro-escala), os efeitos do desmatamento em curso na recião ainda não são detectáveis, uma vez que a maior parte da bacia apresenta florestas intactas e há um intenso processamento dos materiais dentro dos próprios canais dos rios, alterando os sinais biogeoquímicos das mudanças já ocorridas nos usos da terra. Estas mudanças devem ser mais evidentes nos rios das cabeceiras deste vasto sistema de drenagem, os quais constituem o elo mais direto entre os sistemas terrestre e aquático. No entanto, pouco se conhece sobre os efeitos das mudanças no uso da terra no funcionamento e na estrutura desses rios. Estudos em pequenas bacias dos trópicos, abordando os fluxos de matéria orgânica e nutrientes em pequenos rios drenando áreas intactas de floresta, foram efetuados por Chauvel et al. (1987), McDowell \& Asbury (1994), Vegas-Vilarrúbiaet al. (1994), Newbold et al. (1995) e McClain \& Elsenbeer (2001). Por outro lado, os efeitos das atividades humanas nestes ecossistemas, especificamente, o efeito da conversão de florestas em pastagens sobre a biogeoquímica dessas águas, foram descritos por Douglas et al. (1992), Malmer \& Grip (1994), McDowell et al. (1995), Markewitzet al. (2001), Ballester et al. (2003) e Thomas et al. (2004).

Este trabalho teve como objetivos: (1) estabelecer as relações entre os fatores determinantes da bacia de drenagem e a composição biogeoquímica do rio Ji-Paraná. Estes fatores foram categorizados como aqueles diretamente influenciados por atividades humanas (uso do solo e população) e aqueles definidos pelos fatores físicos tais como topografia e solos; (2) definir as consequências biogeoquímicas para aságuas deste rio, associadas com perturbações humanas, em relação à variabilidade ambiental natural.

Este trabalho foi desenvolvido como parte da área denominada "Química da Água e Hidrologia Superficial", do Experimento de Grande Escala Biosfera-Atmosfera na Amazônia (LBA), sendo uma publicação da colaboração internacional entre a Universidade Federal de Rondônia, o Centro de Energia Nuclear na Agricultura - USP, a Universidade de Washington (Seattle, EUA) e o Centro de Ecossistemas (Woods Hole, EUA). As pesquisas foram financiadas pela Fundação de Amparo à Pesquisa do Estado de São Paulo (FAPESP, Processo número 99/01159-4) e pela Agência Americana de Administração Aeronáutica e Espacial (projetos LBA-ECO, ND-03, ND-09 e CD-06).

\section{MATERIAIS E MÉTODOS}

\section{Descrição da área de estudo}

A área de estudo foi a bacia de drenagem do rio Ji-Paraná, localizada na porção leste do estado de Rondônia (Figura 1), em uma das regiões com maiores taxas de desmatamento na Amazônia. Com uma área de drenagem de $75.400 \mathrm{~km}^{2}$ e descarga média anual de $700 \mathrm{~m}^{3} \cdot \mathrm{s}^{-1}$, tem suas nascentes formadas por dois rios: Comemoração e Pimenta Bueno, os quais apresentam baixo grau de alteração do uso e cobertura do solo em seus trechos iniciais. A confluência destes dois rios resulta no rio JiParaná que, ao longo do seu curso, recebe a contribuição de outros 5 tributários principais: Rolim de Moura, Urupá, Jaru, Machadinho e Preto. A medida que o seu canal principal aumenta em ordem, passa, sucessivamente, por áreas com graus de alteração médio, alto e muito alto. Entretanto, no seu trecho final, a partir da entrada do rio Machadinho, passa a drenar uma área com baixo grau de alterção antropogônica. 


\section{ACTA \\ AMAZONICA}

EFEITOS DAS MUDANÇAS DO USO DA TERRA NA BIOGEOQUÍMICA DOS CORPOS D'ÁGUA DA BACIA DO RIO JI-PARANÁ, RONDÔNIA.
Aárea de estudo está localizada em terreno relativamente plano, com alturas que variam de 75 a 600 metros a.n.m, e uma declividade média de 0,62 graus. Os córregos de baixa ordem $\left(1^{\mathrm{a}}\right.$ a $3^{\text {a }}$ ) são dominantes, totalizando um comprimento de 27.497 $\mathrm{km}$. O canal principal do rio tem um comprimento total de 972 km e largura variando de 150 a 500 m, com os canais dos tributários principais variando entre 100 e $400 \mathrm{~m}$. No estado de Rondônia predominam solos antigos, extremamente intemperizados, com baixos níveis de nutrientes, elevada acidez, baixa capacidade de troca catiônica e condições de drenagem pobre. A bacia de drenagem do rio Ji-Paraná é uma exceção a esta regra, com 60\% de sua área contendo solos eutróficos, predominantemente latossolos e podzólicos, com características favoráveis para o desenvolvimento de atividades agrícolas. O clima da reGı̃̃o é caracterizado por temperaturas que variam entre 19 e $33^{\circ} \mathrm{Ce}$ uma precipitação anual em torno de $2500 \mathrm{~mm}$.

\section{Desenho experimental}

Para a obtenção e análise das características ambientais da bacia do rio Ji-Paraná, a mesma foi dividida em setores de acordo com a localização de 14 pontos de medidas da composição biogeoquímica ao longo dos seus principais tributários, organizados de acordo com a morfologia da rede de drenagem e o grau de impacto do uso da terra (Tabela 1, Figura 1). Quatro categorias de impacto de

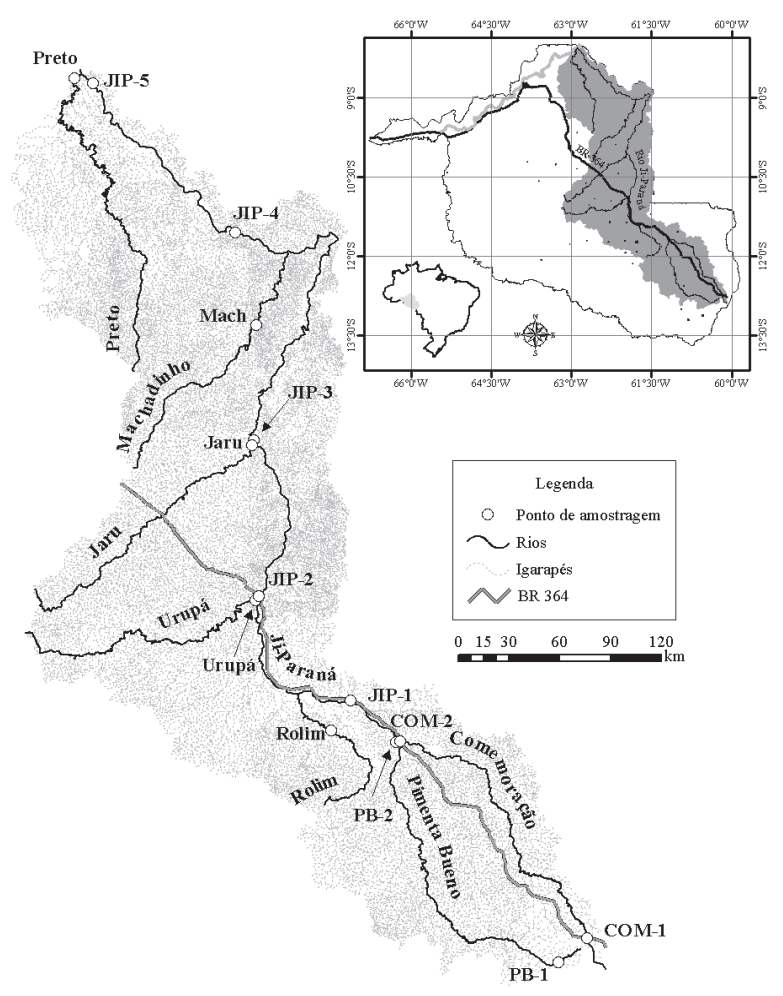

Figura 1 - Localização da bacia de drenagem do rio Ji-Paraná, dos principais tributários e dos pontos de amostragem. uso do solo foram determinadas, em função do percentual de cobertura de pasto: baixo (0 a 15\%); médio (15 a 30\%); alto (30 a $50 \%$ ) e muito alto ( 50 a $75 \%$ ). A ordem de cada rio foi determinada utilizando o método de Strahler (ESRI, 1997). Para determinar o efeito do uso e cobertura do solo da bacia de drenagem na composição biogeoquímica das águas superficiais foram utilizadas apenas duas características da paisagem: a capacidade de troca efetiva (CTE) dos solos para os 20 primeiros centímetros e o uso e cobertura do mesmo. Conforme demonstrado em estudo anterior na região (Ballester et al., no prelo) tais variáveis permitem a identificação e isolamento dos atributos chaves agindo nas unidade de drenagem e que afetam a composição biogeoquímica das mesmas. Dados auxiliares sobre as características físicas (relevo, pedologia e rede de drenagem) e antrópicas (rede de estradas, população, pecuária, projetos de colonização, reservas indígenas e parques nacionais) foram derivadas das bibliotecas digitais georefereneciadas compiladas através do uso do Sistema de Informações Geográficas Arc-GIS (versão 8.1) e de Processamento de Imagem Erdas-Image (versão 8.5) e disponíveis no banco de dados georeferenciados do projeto (juruti.cena.usp.br). Uma descrição detalhada da metodologia para a geração destas bibliotecas pode ser encontrada em Ballester et al. (2003).

\section{Composição biogeoquímica dos sistemas fluviais}

A composição biogeoquímica dos principais tributários da bacia do rio Ji-Paraná foi determinada entre maio de 1999 e dezembro de 2002, a partir de 14 pontos de coletas, totalizando 09 amostragens, e abrangendo as diversas fases da hidrógrafa (Figura 2). As amostras de água para análise química foram coletadas na superfície dos rios e no meio do canal, com o auxílio de uma garrafa de Niskin. Imediatamente após a coleta, alíquotas das amostras foram filtradas em filtros de fibra de vidro pré-calcinados $(0,7 \mu \mathrm{m}, \mathrm{GF} / \mathrm{F})$, acondicionadas em frascos de vidro e preservadas com $\mathrm{HgCl}_{2}$ (concentração final de $\mathrm{Hg}$ de $300 \mu \mathrm{M}$ ), para análise posterior das concentrações de carbono orgânico dissolvido e, em filtros de acetato de celullose $(0,45 \mu \mathrm{m})$, acondicionadas em frascos de polietileno de alta densidade contendo thymol (20 mg para $60 \mathrm{ml}$ ), para as demais análises da

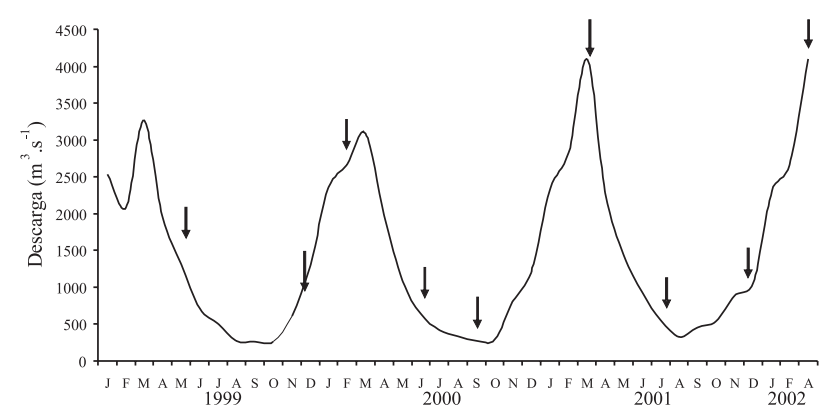

Figura 2 - Variação sazonal da descarga média mensal do rio JiParaná no município de Tabajara, entre janeiro de 1999 e maio de 2002. As setas indicam os estágios da hidrógrafa nos perídos de amostragem. (Fonte: Agência Nacional de Águas). 
Tabela 1 - Identificação dos locais de amostragem e ordem dos rios da bacia do rio JiParaná. O nível impacto de uso do solo foi determinado como uma função do percentual de cobertura de pasto: baixo (0 a 15\%); médio (15 a 30\%); alto (30 a 50\%) e muito alto ( 50 a $75 \%$ ).

\begin{tabular}{lccc}
\hline \hline Sítio/Código & Rio & Ordem & $\begin{array}{c}\text { Nível impacto } \\
\text { de uso do solo }\end{array}$ \\
\hline COM-1 & Comemoração & $3 a$ & Baixo \\
COM-2 & Comemoração & $5 a$ & Médio \\
PB-1 & Pimenta Bueno & $3 a$ & Baixo \\
PB-2 & Pimenta Bueno & $6 a$ & Médio \\
JIP-1 & Ji-Paraná & $6 a$ & Alto \\
ROLIM & Rolim de Moura Moura & $5 a$ & Muito Alto \\
URUPÁ & Urupá & $5 a$ & Muito Alto \\
JIP-2 & Ji-Paraná & $6 a$ & Muito Alto \\
JIP-3 & Ji-Paraná & $6 a$ & Alto \\
JARU & Jaru & $6 a$ & Alto \\
MACH & Machadinho & $5 a$ & Baixo \\
JIP-4 & Ji-Paraná & $7 a$ & Baixo \\
JIP-5 & Ji-Paraná & $7 a$ & Baixo \\
PRETO & Preto & $6 a$ & Baixo \\
\hline \hline
\end{tabular}

indicativo do material de origem que as mesmas drenam, dividindo-se em três grandes categorias (Stallard \& Edmond, 1983): 1) rios com águas com $0<\mathrm{TZ}^{+}<$ $200 \mu$ eq. $\mathrm{l}^{-1}$, drenam materiais mais intensamente intemperizados; 2) rios com águas com $200<\mathrm{TZ}^{+}<450 \mu$ eq..$^{\mathrm{l}^{-1}}$, drenam terrenos ricos em silicatos e 3) rios que apresentam águas com $450<$ $\mathrm{TZ}^{+}<3000 \mu$ eq. $\mathrm{I}^{-1}$, drenam terrenos dominados por carbonatos e têm concentrações elevadas de $\mathrm{Ca}^{2+}, \mathrm{Mg}^{2+} \mathrm{e}$ $\mathrm{SO}_{4}^{2-}$. Utilizando esta classificação, foi possível agrupar as águas do rio Ji-Paraná em três categorias, conforme apresentado na Tabela 2 e ilustrado pelos valores médios de $\mathrm{TZ}^{+}$na Figura 3:

Rios do grupo 1: apresentam valores de $\mathrm{TZ}^{+}$variando entre 25 e $140 \mu$ eq..$^{-1}$, com águas pobres em íons ("águas pretas"). Fazem parte deste grupo as águas dos rios Comemoração, Pimenta Bueno em PB-1, Machadinho e Preto;

Rios do grupo 2: rios com conteúdo composição inorgânica. No campo, foram determinados o pH, com o auxílio de um medidor Orion 250A, a condutividade, com um condutivímetro Amber Science 2052 e a concentração de oxigênio dissolvido e a temperatura, com um medidor Yellow Springs 58. As concentrações dos íons maiores, cálcio, magnésio, sódio, potássio, cloreto e sulfato, e dos nutrientes amônio, nitrato, nitrito e fósforo solúvel reativo foram determinadas por cromatografia líquida, em equipamento Dionex DX-500. As concentrações de carbono orgânico e inorgânico dissolvidos foram determinadas em um TOC-5000 Shimadzu. Todas as concentrações São expressas em M.

\section{Análise dos resultados}

Para testar o efeito das características da paisagem da bacia de drenagem na composição biogeoquímica da águas foi utilizada uma análise de regressão múltipla. As variáveis independentes foram a capaciadade de troca efetiva dos solos e a área coberta por pasto. As variáveis dependentes escolhidas foram: a) traçadores conservativos: condutividade elétria (CE) e as concentrações de $\mathrm{Na}^{+}, \mathrm{Ca}^{2+}, \mathrm{Mg}^{2+}, \mathrm{K}^{+}, \mathrm{Cl}$ e; b) traçadores não conservativos: $\mathrm{PO}_{4}^{3-}$ , nitrogênio inorgânico dissolvido (NID), carbono inorgânico dissolvido (CID) e carbono orgânico dissolvido (COD).

\section{RESULTADOS E DISCUSSÃO}

\section{Padrões I: composição biogeoquímica das águas superficiais}

O somatório dos íons maiores de cargas positivas $\left(\mathrm{TZ}^{+}\right)$ das águas dos rios da Amazônia pode ser utilizado como um iônico intermediários, com valores de $\mathrm{TZ}^{+}$variando entre $275 \mathrm{e}$ $381 \mu$ eq. ${ }^{1-1}$. Este grupo é constituído pelos rios Ji-Paraná e Pimenta Bueno em PB-2;

Rios do grupo 3: são rios de "água branca", com valores de $\mathrm{TZ}^{+}$entre 494 e $729 \mu$ eq. $\mathrm{l}^{-1}$ e conteúdo iônico elevado. Compõem este grupo os rios Rolim de Moura, Urupá e Jarú.

\section{Padrões II: distribuição espacial do uso e cobertura do solo}

A análise do mapa de uso e cobertura do solo da bacia do JiParaná indica que, em 1999, a mesma era composta por $63 \%$ de floresta, $30 \%$ de pasto, $4 \%$ de crescimento secundário e $1.4 \%$ de cerrado. Os centros urbanos, culturas anuais e solos expostos abrangiam menos que $1 \%$ da mesma, respectivamente. Até aquele ano, cerca de $25.700 \mathrm{~km}^{2}$ (ou 35\%) tinham sofrido algum tipo de conversão na cobertura do solo. A distribuição espacial destas modificações era bastante heterogênea, podendo ser identificadas três grande regiões:

1- Nascentes: as mudanças no uso e cobertura do solo foram mais substanciais no setor COM-1, onde $60 \%$ da floresta original tinha sido suàstituída por culturas anuais (43\%) ou pastagens (17\%). O setor PB-1 permaneu praticamente intacto, com $90 \%$ de sua área coberta por floresta. Amedida que o rio avança nos setores seguintes (COM-2 e PB-2), este padrno inverte-se. O Pimenta Bueno tinha $33 \%$ de pasto e mais da metade do setor era ainda coberto por vegetação nativa, incluindo a floresta (59\%) e cerrado (0,4\%). Já no setor COM-2, a floresta nativa cobria $66 \%$ daárea, enquanto o pasto extendia-se em $29 \%$ da mesma. Neste setor encontra-se a cidade de Vilhena, um dos centros urbanos mais importantes da bacia, o qual ocupava, em 1999, 2,4\% daárea do setor. 


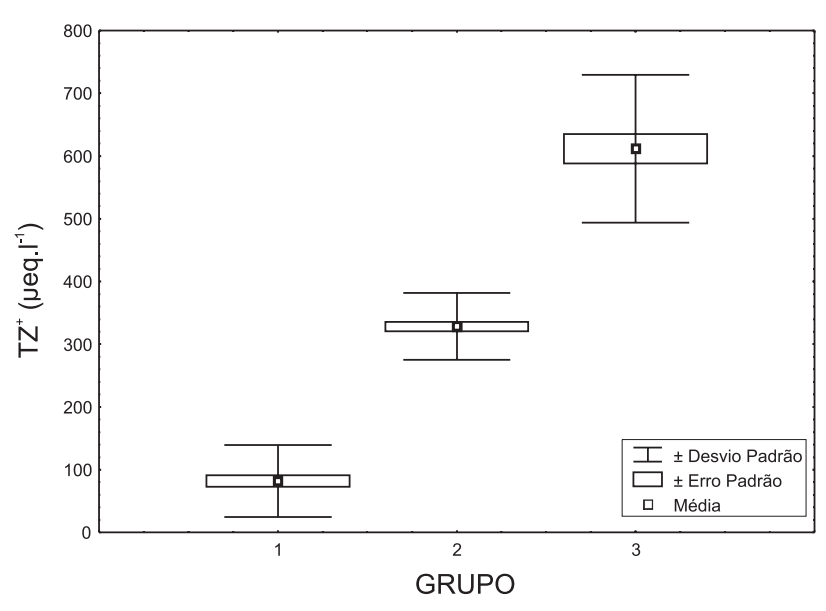

Figura 3 - Valores médios do somatório de cargas positivas $\left(\mathrm{TZ}+\right.$, em $\mu$ eq. $\left.\mathrm{L}^{-1}\right)$ nas águas superficiais da bacia do rio JiParaná. Rios do grupo 1 apresentam valores de TZ+ entre 25 e 200, rios do grupo 2 valores entre 201 e 450 e rios do grupo 3 valores entre 451 e $653 \mu$ eq.L ${ }^{-1}$.

2- Área central:composta pelos setores JIP-1, 2, 3, Rolim, Urupá e Jaru. Entre os mesmos, JIP-1 apresentava as mudanças mais pronunciadas e a maior área de pastagem da bacia. Da área total do setor, $69 \%$ tinha sido convertida em pasto e apenas $28 \%$ da floresta original foi deixada intacta. Um padrão bastante semelhante foi observado em Rolim de Moura, com valores de $66 \%$ de pasto e $26 \%$ de floresta. JIP-2, JIP-3 e Jaru apresentaram também similaridades em termos da área de pastagens (56\%, $53 \%$ e $53 \%$, respectivamente) e floresta (36\%, 37\% e 36\%, respectivamente). No setor de Urupá a floresta ocupava $50 \%$ da área e o pasto $43 \%$.

3-Área final do percurso do rio: nesta região observa-se uma reversão no padrão de uso e cobertura. O pasto torna-se menos comum e a paisagem passa a ser dominada pela vegetação nativa, incluindo florestas e cerrados. No setor Machadinho, apenas 22\% da área era coberta por pasto, permanecendo ainda $68 \%$ da floresta intacta. No setor JIP-4, $8 \% \mathrm{da}$ área eram cobertos por pasto e $87 \%$ por floresta. Neste setor ocorre o maior fragmento de cerrado da bacia, cobrindo $221 \mathrm{~km}^{2}$. A porção final da bacia do rio Ji-Paraná era a menos perturbada. JIP- 5 tinha menos de $1 \%$ de sua área coberta por pasto, $89 \%$ por floresta e $2 \%$ por cerrado. A parte ocidental da bacia foi ligeiramente modificada, o setor Preto tinha $4 \%$ de pasto e $87 \%$ de floresta.

\section{Processos: fatores responsáveis pelas mudanças no uso da terra}

Os estudos das causas do desmatamento em áreas tropicais têm demonstrado que a imigração e o crescimento natural da população dirigem a expansão das lavouras e pastagens em $47 \%$ dos casos estudados na America Latina. O crescimento das pastagens associa-se aos processos de colonização, tanto planejados, quanto espontâneos, dos agricultores (Geinst \&
Lambin, 2002). No caso específico da Amazônia brasileira, uma das principais causas do desmatamento tem sido a substituição da floresta tropical em áreas de pastagem para a pecuária. A principal prática de conversão consiste na derrubada e queima, mecanismo que incialmente enriquece a superfície do solo em cátions provenientes da biomassa vegetal queimada e transferida para o mesmo na forma de cinzas (Kauffman et al., 1998; Markewitz et al., 2001). Este tipo de processo é comumente observado em Rondônia, onde a ocupação resultou em uma pecuária bovina extensiva como principal atividade econômica do Estado (Marconato, 2002).

Entre 1970 e 1990, o estado de Rondônia teve um impulso no desenvolvimento e ocupação (Figura 4), aumentando a população de 111.064 pessoas residentes em 1970 para 1.379.787 pessoas em 2000 (IBGE, 2003). A ocupação ocorreu a partir da região centro-leste, desenvolvendo intensamente a atividade pecuária bovina nesta região (Figura 5), associada à perda de $24,4 \%$ da cobertura florestal nativa do estado de Rondônia (Brasil, 2002). As mudanças no uso do solo mais intensas ocorreram na região central da bacia do rio Ji-Paraná, onde a maioria dos projetos de colonização foram estabelecidos e onde concentrou-se a abertura de estradas (Ballester $\mathrm{et} \mathrm{al}$., 2003). Áreas extensas de florestas nativas foram submetidas a mudanças rápidas, resultando em um padrão do tipo "espinha de peixe". Em 1999, 72\% do total da área desmatada na bacia estava localizada em áreas situadas até 20 quilômetros de distância de uma estrada principal. No mesmo ano, $63 \%$ da área total desmatada na bacia estava situada nos limites de um projeto de colonização e apenas $25 \%$ da cobertura florestal original permanecia intacta nos mesmos.

Outro fator importante na determinação dos padrões espaço-temporais de dispersão das pastagens, o qual tem recebido menos atenção, são as propriedades do solo. De modo general, o estado de Rondônia apresenta solos principalmente velhos e altamente intemperizados, que perderam grande parte de seus reservatórios de minerais. O potencial agrícola da maioria destes solos é limitado por fatores tais como níveis baixos de nutrientes, acidez elevada, baixa capacidade de troca e condições pobres de drenagem (Diegues, 1999). A bacia do rio de Ji-Paraná é uma exceção a este padrão geral, com sua maior parte (em torno de $60 \%$ ) coberta por solos eutróficos que são favoráveis Às atividades agrícolas (IBGE, 1993). Na bacia de drenagem do rio Ji-Paraná, pastagens estão espacialmente associadas a solos com níveis de nutrientes mais elevados da parte central da bacia (JIP-1, 2 e 3, Rolim, Urupá e Jaru em 1993). Da área total coberta com pasto, $90 \%$ estavam situados em solos eutróficos e somente $10 \%$ nos distróficos. Nas áreas com florestas e cerrados, o oposto ocorre, embora as porcentagens de cada tipo do solo sejam similares. Aproximadamente $48 \%$ da vegetação natural estava situada em solos eutróficos e $52 \%$ em solos distróficos. Solos com saturação de bases mais elevados estão espacialmente associados a áreas com maior intensidade de uso agrícola, relação esta estatisticamente significativa $\left(\mathrm{r}^{2}=0,70998, \mathrm{p}<0,001\right.$; Ballester et al., 2003). 


\section{ACTA AMAZONICA}

EFEITOS DAS MUDANÇAS DO USO DA TERRA NA BIOGEOQUÍMICA DOS CORPOS D'ÁGUA DA BACIA DO RIO JI-PARANÁ, RONDÔNIA.

\section{De padrões a processos}

Em termos das conseqüências biogeoquímicas para o rio, a distribuição espacial heterogênea do desmatamento e das propriedades do solo ao longo da bacia se traduzem em sinais diferentes (Victoria et al., 2000; Richey et al., 2001). Os setores do rio com solos com conteúdo de nutrientes mais baixos tiveram também águas com um conteúdo iônico menor, enquanto solos com concentrações de nutrientes mais elevados são drenados por águas mais ricas em íons. Para a maioria dos parâmetros analisados, a distribuição espacial dos três grupos iônicos descritos anteriormente é consistente com a distribuição espacial das áreas de pasto. A análise de regressão múltipla mostrou que as características da qualidade de água do rio têm uma relação estatisticamente significativa com a cobertura de pasto (Ballester et al., 2003), a qual explica a maior parte da variabilidade observada para todos os parâmetros, com exceção do cálcio e do magnésio. Nestes casos, CTE e a pastagem foram bons previsores das concentrações de cátions observadas.

Portanto, os resultados obtidos demonstram que o pasto é um dos fatores determinantes na composição química das águas superficiais dos rios estudados. Em termos de distribuição espacial, valores mais elevados para todos os íons analisados estiveram associados com os setores dominados pelo pasto. As concentrações destes elementos mostraram um aumento consistente a medida que o rio drena áreas com
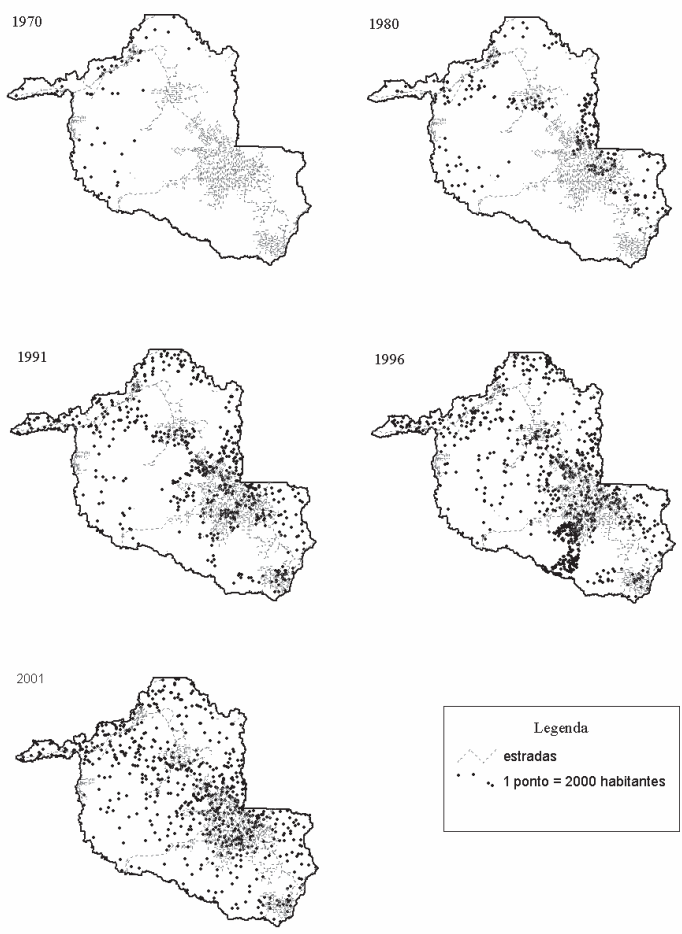

Figura 4 - Distribuição espaço-temporal da população no estado de Rondônia entre 1970 e 2001. Fonte: Instituto Brasileiro de Geografia e Estatística, IBGE. um aumento na proporção de pasto. As concentrações mais elevadas foram encontradas na parte central da bacia, onde as áreas de pasto atingem um máximo. A medida que o rio entra nos trechos finais de drenagem, a floresta domina a paisagem e as concentrações caem.

A porcentagem da área da bacia coberta pelo pasto foi consistentemente o melhor parâmetro para prever a condutividade elétrica (com um coeficiente de correlação, $\mathrm{r}^{2}$, de $0,872)$ e as concentrações de sódio $\left(\mathrm{r}^{2}=0,754\right)$, cloreto $\left(\mathrm{r}^{2}=\right.$ $0,692)$ e potássio $\left(r^{2}=0,626\right)$. Para o cálcio, a Capacidade de Troca Efetiva $\left(r^{2}=0,538\right)$ e o pasto $\left(r^{2}=0,502\right)$ explicaram a maior parte da variabilidade observada. O mesmo tipo de padrno foi encontrado para o magnésio $\left(r^{2}=0,498\right.$ e 0,502 , respectivamente). Estes resultados indicam que a variabilidade espacial natural das características dos solo, tal como a Capacidade de Troca Efetiva, é um fator determinante importante da composição biogeoquímica dos elementos conservativos das águas superficiais. Markewitzet al. (2001) demonstraram que, ao contrário do que ocorre em rios que drenam áreas de climas temperados e com rochas recentes, em Paragominas, PA, onde as rochas SÃo antigas e muito intemperisadas, a química das águas daqueles igarapés mostra estreita relação com a composição dos solos. Isto significa que os aportes provenientes deste compartimento são relevantes para a biogeoquímica destes sistemas fluviais e que, mudanças no seu uso/cobertura devem apresentar reflexos nestas águas. Elsenbeer et al. (1999)

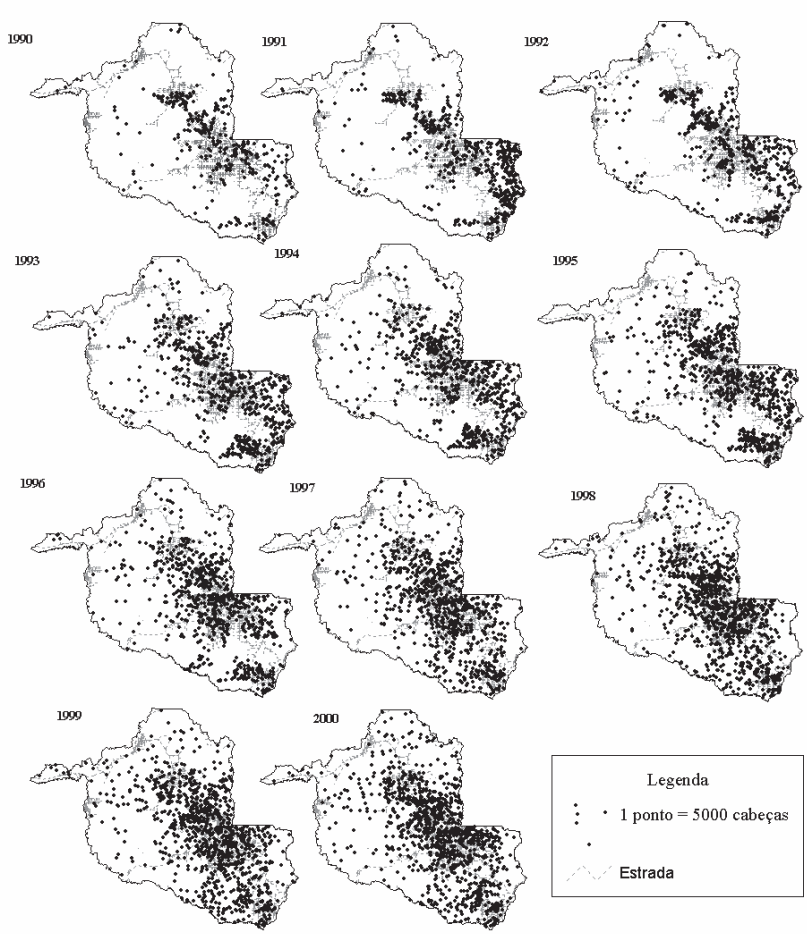

Figura 5 - Distribuição espaço-temporal da pecuária (número de cabeças de gado) no estado de Rondônia entre 1990 e 2000. Fonte: Instituto Brasileiro de Geografia e Estatística, IBGE. 


\section{ACTA \\ AMAZONICA}

EFEITOS DAS MUDANÇAS DO USO DA TERRA NA BIOGEOQUÍMICA DOS CORPOS D'ÁGUA DA BACIA DO RIO JI-PARANÁ, RONDÔNIA. mostraram que a compactação dos solos em pastagen aumenta o potencial para a ocorrência de um fluxo lateral, que, quando combinada com o manejo inadequado do pasto (Kauffman et al., 1998; Dias-Filho et al., 2001) ou com o escoamento superficial das excreções do gado (fezes e urina), poderiam também influenciar a composição química destas águas.

ACTE é uma medida da habilidade do solo em reter cátions (Parker \& Rae, 1998) e, apesar das diferenças na CTE relatadas na literatura para pastos de idades diferentes na Amazônia (Moraes et al., 1996), nenhuma tendência estatisticamente significativa foi identificada até o momento, em termos de perdas ou ganhos dramáticos nos estoques de nutrientes dos solos superfíciais, quando solos de pastos foram comparados com àqueles de florestas na Amazônia (Kauffman et al., 1995; Moraes et al., 1996; Kauffman et al., 1998). Além disso, quando as áreas do pasto são instaladas, um aumento nas concentrações de $\mathrm{Ca}^{2+}, \mathrm{Mg}^{2+} \mathrm{e} \mathrm{K}^{+}$na camada superior do solo $(0 \mathrm{a} 5 \mathrm{~cm}$ ) foi observada durante os primeiros 1 a 5 anos. Este aumento foi atribuído ao efeito da deposição de cinzas. Entretanto, decréscimos na disponibilidade de nutrientes no solo devido à lixiviação tornaram-se significativos após 6 anos da instalação do pasto (Moraes et al., 1996; Kauffman et al., 1998). Este poderia ser o caso da maioria dos pastos na bacia do rio de JiParaná, onde o uso do fogo é uma prática difundida da eliminação dos resíduos do corte e o manejo do pasto.

Contudo, a importância relativa de cada componente da paisagem na composição química das águas superficiais da Amazônia deve ainda ser melhor avaliada em função da escala de trabalho. De acordo com Thomas et al. (2004), a biogeoquímica de elementos não conservativos, como nitrogênio e carbono, dos setores de primeira ordem de igarapés da Fazenda Nova Vida (Rondônia), altera-se drasticamente quando a floresta é suàstituída por pastagens. Tal comportamento pode ser atribuído ao fato de nos igarapés de baixa ordem $\left(1^{\mathrm{a}} \mathrm{a} 2^{\mathrm{a}}\right)$ da região ser comum o desenvolvimento da gramínea Paspallum dentro dos canais, o qual altera o metabolismo destas águas, em função da respiração das raízes e/ou decomposição desta matéria orgânica lábil. Nestas condições, o ambiente torna-se mais anaeróbio, favorecendo os processos e ciclagem associados a estas condições. Porém, à medida em que os rios evoluem para setores de ordem superior (já a partir de setores de $3^{\mathrm{a}}$ ordem) estes sistemas fluviais apresentam características mistas, muitas vezes similares àquelas dos trechos florestados, mesmo que tenham a maior parte de suas bacias de drenagem cobertas com pastagens.

Este padrão não se repete na bacia doJi-Paraná, na qual foi observada uma correlação estatisticamente significativa entre a área coberta por pastagens e as concentrações de $\mathrm{PO}_{4}^{3 \cdot}\left(\mathrm{r}^{2}=\right.$ $0,78, \mathrm{p}<0,01)$, nitrogênio inorgânico dissolvido (NID) $\left(\mathrm{r}^{2}=\right.$ $0,52, \mathrm{p}<0,01)$, carbono inorgânico dissolvido (CID) $\left(\mathrm{r}^{2}=0,81\right.$, $\mathrm{p}<0,01)$ e carbono orgânico dissolvido (COD) $\left(\mathrm{r}^{2}=0,51, \mathrm{p}<\right.$ $0,01)$. Estes resultados indicam que as mudanças observadas na micro-escala constituem "sinais biogeoquímicos" gerados pelo processamento do material nas margens dos rios. Amedida em que os rios evoluem para ordens superiores, na meso escala, os "sinais biogeoquímicos" persistentes nos canais fluviais estão associdados às características da bacia de drenagem (incluindo solos e uso da terra), ao invés daqueles gerados nas zonas margens, e passam a ser os determinantes da dinâmica destes sistemas.

Simultaneamente, o fato do pasto ter sido melhor previsor para nitrogênio, fósforo e carbono orgânico é também uma indicação que as mudanças na composição da paisagem (uso da terra) têm um impacto potencial na biogeoquímica das águas do rio. De acordo com estes resultados, a substituição da floresta pelo pasto pode resultar em mudanças significativas na concentração de nutrientes. De acordo com nossa análise de regressão, um aumento de $10 \%$ na área de pasto no setor do baixo Ji-Paraná (JIP-5) poderia resultar em um aumento de três vezes na concentração de fosfato, e uma vez e meia na concentração de NID, COD e CID na água.

Detectar impactos humanos em um sistema fluvial é desafiador, em função dos diversos componentes biológicos, químicos, hidrológicos e geofísicos que devem ser avaliados (Gergel et al., 2002). Modelos empíricos têm que ser desenvolvidos para permitir um conhecimento maior do funcionamento destes ecossistemas e efetuar simulações e previstes de eventos futuros. Nossos resultados permitem, até o momento, concluir que com base nos padrões do estabelecimento do pasto observados neste estudo e no tipo de solos da bacia, não é provável que o desmatamento ocorra nas regiões das cabeceiras, devido Às características arenosas dos solos desta região. As áreas que devem causar uma maior preocupação em termos ambientais devem se concentrar, preferencialmente, nos setores à jusante do rio, onde a floresta ocupa áreas potencialmente agriculturáveis de solos mais ricos.

Em termos de funcionamento do ecossistema amazônico, apesar dos efeitos das mudanças observadas no uso do solo não serem ainda detectáveis na macro-escala (bacia amazônica como um todo), a disrupção da estrutura e funcionamento dos ecossistemas é detectável na micro e meso escalas, com alterações significativas na ciclagem de nutrientes nos ecossistemas fluviais.

\section{BIBLIOGRAFIA CITADA}

Ballester, M.V.R.; Victoria, D. DE C.; Coburn, R.; Krusche, A.V.; Victoria, R.L.; Richey, J.E.; Logsdon, M.G.; Mayorga, E.; Matricardi, E. 2003. A Remote Sensing/GIS-based physical template to understand the biogeochemistry of the JiParaná River Basin (Western Amazônia). Remote Sensing of the Environment, 87(4):429-445.

Brasil, 2002. INPE. Monitoring of the Brazilian Amazon forest by satellite 1999-2000. National Institute for Space Research. São José dos Campos, SP, Brazil.

Chauvel, A.; Lucas, Y.; Boulet, R. 1987. On the genesis of the soil mantle of the region of Manaus, Central Amazonia, Brazil. Experientia, 43:234-241.

Dias-Filho, M.B.; Davidson, E.A.; Reis, de C. C.J. 2001. Linking biogeochemical cycles to cattle pasture management and sustainability in the Amazon basin. In: McClain, M.E.; Victoria, R.L. and Richey, R.E. Eds. The biogeochemistry of the Amazon basin. Oxford University Press. Oxford. p. 84-105. 
Douglas, I., T., et al. 1992. The impact of selective commercial logging on stream hydrology, chemistry and sediment loads in the Ulu Segama Rain Forest, Sabah. Philosophical Transactions of the Royal Society of London B, 335:397-406.

ERDAS-IMAGINE. 1999.User's manual.459pp.

ESRI, 1997. Arc-Info version 7.3. (software) Redlands, California.

Gash, J.H.C.; Nobre, C.A.; Roberts, M.J.; Victoria, R.L.1996. Amazonian deforestation and Climate. Wiley and Sons, New York, USA. 612pp.

Geinst, H. J.; Lambin, E. F. 2002. Proximate Causes and Underlying Driving Forces of Tropical Deforestation. BioScience, 52 (2): 143-149.

Gergel, S.A.; Turner, M.G.; Miller, J.R.; Melack, J.M. and Stantely, E.H. 2002. Landscape indicators of human impacts to riverine systems. Aquatic Science, 64: 118-128.

Hunsaker C.T.; Levine D.A. 1995. Hierarchical Approaches to the Study of Water Quality in Rivers. BioScience, 45, 193-203.

I.B.G.E. Instituto Brasileiro de Geografia e Estatística.1993. Recursos naturais e meio ambiente. Uma visão do Brasil. IBGE. Rio de Janeiro. Brasil. 154pp.

Junk, W. J. 1997. General aspects of floodplain ecology with special reference to Amazonian floodplains. In: Junk, W. J. (ed.) The Central Amazon Floodplain. SpringerVerlag, Berlin. p. 3-20.

Kauffman, J.B.; Cummings, D.L.; Ward, D.E. and Babbitt, R. 1995. Fire in the Brazilian Amazon: 1. Biomass, nutrient pools, and losses in slashed primary forest. Oecologia, 104: 397- 408.

Kauffman, J.B.; Cummings, D.L. and Ward, D.E. . 1998. Fire in the Brazilian Amazon: 2. Biomass, nutrient pools, and losses in cattle pastures. Oecologia, 113: 415-427.

Leopoldo, P. R.; Franken, W.; Salati, E. and Ribeiro, M. N. 1987. Towards a water balance in the Central Amazonian region. Experientia, 43:222-233.

Lesack, L. F. W. \& Melack, J. M. 1995. Flooding hydrology and mixture dynamics of lake water derived from multiple sources in an Amazon floodplain lake. Water Resources Research, 31:329-345.

Malmer, A. and H. Grip. 1994. Converting tropical rainforest to forest plantation in Sabah, Malaysia. 2. Effects on nutrient dynamics and net losses in streamwater. Hydrological Processes, 8:195-209.

Marconato, R. 2002. Análise da ocupação econômica do estado de Rondônia nos anos 90 segundo o modelo de Krugman.FE/ESALQ/USP, CENA/USP. Monografia. 108p. Il.

Markewitz, E; Davidson, E.A.; Figueiredo, A.R. de O.; Victoria, R. L. and Krusche, A.V. 2001. Control of cation concentrations in stream waters by surface soil processes in an Amazonian watershed. Nature, 410: 802-805.

McClain, M E. e Elsenbeer, H. 2001. Terrestrial inputs to amazon streams and internal biogeochemical processing. In: The biogeochemistry of the Amazon basin. McClain, M.E.; Victoria, R.L. and Richey, R.E. Eds. Oxford University Press. Oxford p. 185-208.
McDowell, W. H.; C. E. Asbury. 1994. Export of carbon, N and major ions from three tropical montane watersheds. Limnology and Oceanography, 39:111-125.

McDowell, W. H., et al. 1995. Export of nutrients and major ions from Caribbean catchments. J. N. Am. Benthol. Soc, 14(1):12-20.

Meixner, F.X.; Eugster, W. 1999. Effects of landscape pattern and topography in emissions an transport. In Tenhunen, J.D. and Kabat, P. Eds. Integrating bydrology, ecosystem dinamics, and biogeochemistry in complex landscapes. Wiley and Sons, New York. p. 147- 175.

Melack, J. M.; T. R. Fisher. 1990. Comparative limnology of tropical floodplain lakes with an emphasis on the central Amazon. Acta Limnologica Brasiliensia, 3:1-48.

Meyer, J. L.; McDowell, W. H.; Bott, T. L.; Elwood, J. W.; Ishizaki, C.; Melack, J. M.; Peckarsky, B. L.; Peterson, B. J.; Rublee, P. A. 1988. Elemental dynamics in streams. Journal of the North American Benthological Society, 7:410-432.

Moraes, J.F.L.; Volkoff, B.; Cerri, C.C. and Bernoux, M. 1996. Soil properties under Amazon forest and changes due to pasture installation in Rondônia, Brazil. Geoderma, 70: 63-81.

Newbold, J. D., et al. 1995. Concentration and export of solutes from six mountain streams in northwestern Costa Rica. $J$. N. Am. Benthol. Soc., 14:21-37.

Parker, A.; Rae, J.E. 1998. Environmental interactions of clays. Clays and the environment. Springer-Verlang, Berlin, Germany. 271 pp.

Richey, J. E., et al. 1980. Organic carbon: oxidation and transport in the Amazon River. Science, 207:1348-1351.

Richey, J. E., et al. 1990. Biogeochemistry of carbon in the Amazon River. Limnology and Oceanography, 35:352-371

Richey, J. E., et al. 1991. The biogeochemistry of a major river system: The Amazon case study. In: Degens et al. (eds.) Biogeochemistry of major world rivers. John Wiley and Sons, New York. p. 57-74.

Richey, J. E.; R. L. Victoria. 1993. C, N, and P export dynamics in the Amazon River. In: Wollast et al. (eds.) Interactions of $C$, $N, P$, and $S$ biogeochemical cycles and global change. Springer-Verlag, Berlin. p. 123-140.

Richey, J.E.; Krusche, A.V.; Deegan, L.; Ballester, M.V.R.; Biggs, T., Victoria, R.L.2001. Land use change and the biogeochemistry of river corridors in Amazon. Global Change News Letter, 45:19-22.

Richey, J.E.; Melack, J.M.; Aufdenkampe, A.K.; Ballester, M.V.; Hess, L.L. 2002. Outgassing from Amazonian rivers and wetlands as a large tropical source of atmospheric $\mathrm{CO}_{2}$. Nature, 416: 617-620.

Salas, W. A.; Ducey, M. J.; Rignot, E.; Skole, D. 2002. Assessment of JERS-1 SAR for monitoring secondary vegetation in Amazonia: I. Spatial and temporal variability in backscatter across a chrono-sequence of secondary vegetation stands in Rondônia. International Journal of Remote Sensing, 23(7): 1357-1379. 
Sharpley, A. N.; Hedley, M. J.; Sibbesen, E.; Hillbricht-Ilkowska, A.; House, W. A.; Ryskowski, I. 1995. Phosphorus transfers from terrestrial to aquatic systems. In $\mathrm{H}$. Tiessen (ed.), Phosphorus in the Global Environment. SCOPE 54. John Wiley. NY. p. 171-200.

Sioli, H. 1984. The Amazon: Limnology and landscape ecology of a mighty tropical river and its basin. Dr. W. Junk Publishers, Boston. 763pp.

Sippel, S.J., et al. 1994. Determination of inundation area in the Amazon River floodplain using the SMMR $37 \mathrm{GHz}$ polarization difference. Remote Sensing of Environment, 48: 70-76.

Stallard, R. F.; J. M. Edmond. 1983. Geochemistry of the Amazon. 2. The influence of geology and weathering environment on the dissolved load. Journal of Geophysical Research, 88:9671-9688.

Stallard, R. F.; J. M. Edmond. 1987. Geochemistry of the Amazon. 3. Weathering chemistry and limits to dissolved input. Journal of Geophys. Res., 92:8293-8302.

Swank, W. T.; D. A. Grossley (eds.). 1988. Forest Hydrology and Ecology at Coweeta. Springer-Verlag, NY. 66: 339-357.
Thomas, S.; Neill, C.; Deegan, L.; Krusche, A.V. and Victoria, R.L. Influences of land use and stream size on particulate and dissolved materials in a small Amazonian stream network. Biogeochemistry, 68:135-151.

Vegas-Vilarrúbia, T., et al. 1994. Small catchment studies in the tropical zone. In B. Moldan and J. Cerný (eds.). Biogeochemistry of Small Catchments: A Tool for Environmental Research. SCOPE 51, J. Wiley, NY. p.343-360

Victoria, R.L.; Richey, J.E; Bernardes, M.; Ballester, M.V.R.; Gomes, B.M.; Krusche, A.V.; Mayorga, E. 2000. "Effects of land use changes in the biogeochemistry of Ji-Paraná river, a meso-scale river in the state of Rondônia, southern Amazon". Proceedings of the LBA Experiment in Amazonia First Scientific Conference. Belém, Pará - Brazil. June 25-28.

Vitousek, P. M.; Melillo, J. 1979. Nitrate losses from disturbed ecosystems. Science, 204:469-474.

RECEBIDO EM 05/10/2003

ACEITO EM 14/06/2005 
